\title{
ENCYSTATION COMMITMENT IN GIARDIA DUODENALIS: A LONG AND WINDING ROAD
}

\author{
ARGÜELLO-GARCIÁ R.*, BAZÁN-TEJEDA M.L.* \& ORTEGA-PIERRES G.*
}

\section{Summary :}

Cholesterol and bile salts are relevant modulators of Giardia encystation. Although several molecules within signaling cascades have been identified, and changes in their expression observed during giardial encystation, their underlying interactions leading to expression of cyst wall markers (CWPs and precursors of the GalNAc homopolymer) are not well defined. Recent experimental data and the completion of the Giardia Genome Project Database (GiardiaDB) allow us now to consider the role of bile salts as "natural stimuli" and the potential involvement of a Raf/MEK/ERK pathway mediating cholesterol-regulated expression of cyst-specific genes. These new findings may provide promising targets for diagnostics, drug design and prophylactic intervention against giardiasis.

KEY WORDS : Giardia duodenalis, encystation, cyst wall, cholesterol, bile salts, cholesterol receptor, SREBP, cell signaling, protist.

\section{INTRODUCTION}

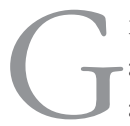
iardiasis is a leading diarrhoeal illness in humans - and a common enteric infection in companion animals and livestock. WHO estimates an incidence of 2-7\% in developed countries. In Asia, Africa and Latin America three billion people live at risk of the disease and this high prevalence results in one billion cases, contributing to some 2.5 million deaths annually from diarrheal disease. In 2004 giardiasis was included in the 'Neglected Disease Initiative'. In addition, Giardia is considered a category B agent of bioterrorism by CDC and it is the most widespread protist-associated diarrhoea with 200 million symptomatic individuals worldwide at any given time (WHO, 2006). The causal agent, Giardia duodenalis (syn. G. intestinalis, G. lamblia) has two stages: the pathogenic, vegetative trophozoite and the infective, quiescent cyst. The

\footnotetext{
* Departamento de Genética y Biología Molecular, Centro de Investigación y de Estudios Avanzados del I.P.N., Av. Instituto Politécnico Nacional 2508, Col. San Pedro Zacatenco, 07360 Mexico City, México.

Correspondence: Guadalupe Ortega-Pierres.

Tel.: (52) 5557473331 - Fax: (52) 5557473392.

E-mail: gortega@cinvestav.mx
}

Résumé : L'ENGAGEMENT DE GIARDIA DUODENALIS DANS L'ENKYSTEMENT : UN PARCOURS LONG ET SINUEUX

Le cholestérol et les sels biliaires sont des modulateurs impliqués dans l'enkystement de Giardia. Bien que plusieurs molécules de signalisation en cascades et leurs changements d'expression lors de l'enkystement aient été étudiés, les interactions sous-jacentes, conduisant à l'expression des marqueurs de la paroi kystique (CWPs et précurseurs du GalNac homoplolymère) ne sont pas bien définies. Des données expérimentales récentes et l'achèvement du projet de séquençage du génome de Giardia (Giardia DB) nous permettent actuellement d'étudier le rôle des sels biliaires en tant que "stimuli naturels" et l'implication potentielle d'une voie Raf/MEK/ERK dans la médiation par le cholestérol de l'expression de gènes spécifiques du kyste. Ces nouvelles avancées peuvent permettre de proposer des cibles prometteuses pour le diagnostic, et de nouvelles pistes pour la conception de médicaments et de méthodes prophylactiques contre la giardiose.

MOTS CLÉS : Giardia duodenalis, enkystement, paroi kystique, cholestérol, sels biliaires, récepteur du cholestérol, SREBP, signalisation cellulaire, protiste.

trophozoite-to-cyst conversion process (encystation) of this parasite represents a primitive response to cellular stress. Likewise Giardia is a relatively simple eukaryote with reduced/cryptic structures such as mitosomes, Golgi-like apparatus and nucleolus (Tovar et al., 2003; Luján \& Touz, 2003; Jiménez-García et al., 2008) coupled to a compact genome encoding a limited proteomic repertoire (Morrison et al., 2007). Nevertheless Giardia is considered as the most highly evolved and adaptedto-parasitism diplomonad on the basis of morphogenetic criteria (Keeling \& Brugerolle, 2006). The reproduction of the giardial life cycle in vitro (Gillin et al., 1987, 1989), the rodent models of giardiasis and the Giardia Genome Project Database (GiardiaDB at http://www.giardiadb.org/ giardiadb/) are useful platforms from which to analyze Giardia en/excystation processes.

\section{FACTORS INVOLVED IN ENCYSTATION INDUCTION}

Tardia encystation is a multifactorial process that requires four defined factors in the extracellular medium as defined by the following 
protocols using serum-supplemented TYI-S-33 medium for cyst production: a) deprivation of specific nutrients as cholesterol (Luján et al., 1997); b) inhibition of agonist-receptor interaction by antibodies directed against the cholesterol receptor (CR) with induction of a sterolresponse element binding protein (SREBP) homolog (Kaul et al., 2001; Worgall et al., 2004); c) physicochemical factors viz., gas infusion [N2/O2], (Sterling et al., 1988); and d) host-derived factors including bile or bile components (primary bile salts viz., micellar glycocholate and fatty acids viz., myristic acid) at slightly alkaline $\mathrm{pH}$ (Gillin et al., 1987, 1988). Of these protocols, a high concentration of bile $(5 \mathrm{mg} / \mathrm{ml})$ at $\mathrm{pH} 7.8$ is widely used although encystation was also induced in the presence of extracellular cholesterol (Kaul et al., 2001; Sterling et al., 1988) and/or in the absence of bile components (Luján et al., 1997; Sterling et al., 1988). Indeed cholesterol and bile components are relevant modulators of giardial encystation and their role in this process is discussed herein.

\section{MOLECULAR AND STRUCTURAL MARKERS OF GIARDIAL ENCYSTATION}

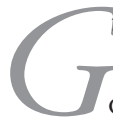
Tardia encystation entails a decrease in adhesion, reduced metabolic rate and multiplication of the pear-shaped trophozoite resulting in rounding-up, disassembly of the ventral disk, internalization of flagella, encasement in a protective envelope, the cyst wall (CW), and entering into cryptobiosis (a state still metabolically active but reduced to $20 \%$ of that of trophozoites). CW is a hallmark of encystation and plays a critical role in cysts since it resists a plethora of environmental stresses, excludes most small molecules and aids in sensing the stimuli for excystation. The CW matrix is composed of an inner twolayered membranous and an outer protofilamentous layer (CWF) 0.3-0.5 $\mu \mathrm{m}$ thick with filaments $7-15 \mathrm{~nm}$ in diameter. The filaments have two main components: $63 \%$ by weight of an unique $\beta 1,3$-linked (GalNAc) homopolymer (Jarroll et al., 1986; Manning et al., 1992; Gerwig et al., 2002) and $37 \%$ by weight of at least four different embedded structural polypeptides: three are leucine-rich repeats (LRR)-containing proteins (CWPs 1-3) and the other is a cysteine-rich and variant surface protein (VSP)-like molecule named HCNCp (Mowatt et al., 1995; Luján et al., 1995; Sun et al., 2003; Davids et al., 2006).

The biosynthesis of the GalNAc glycopolymer is carried out by two processes: one consists of a cytosolic five-step enzymatic pathway that is developmentally induced/transcriptionally regulated (López et al., 2003) and converts fructuose-6-phosphate diverted from glycolysis into uridine diphosphate (UDP)-GalNAc, the sac- charide precursor of the $[\operatorname{GalNAc}(\beta 1 \rightarrow 3) \operatorname{GalNAc}(\beta 1 \rightarrow 3)] \mathrm{n}$ homopolymer (Jarroll et al., 2001). The first enzyme of this pathway, glucosamine-6-phosphate-isomerase (G6PI), is reversible since it exhibits aminase/deaminase activities (Steimle et al., 1997) and the fourth enzyme (UDP-GlcNAc pyrophosphorylase) is a putative ratelimiting enzyme allosterically activated in the anabolic direction by glucosamine-6-phosphate levels (Bulik et al., 2000). The other process is the synthesis of the CW polysaccharide, where a particle-associated transferase activity tentatively referred to as cyst wall synthase (CWS) has been reported (Craig \& Jarroll, 2004). Whether one or various enzymes are responsible for CWS activity is yet to be defined.

All CWPs are developmentally and coordinately expressed, co-sorted to electron-dense secretory granules named encystation-specific vesicles (ESV), co-transported and co-localized exclusively to CWF (Fig. 1). HCNCp is detected in the nuclear envelope of trophozoites, ESV, CWF and the cell body within mature cysts. Each CWP is encoded by a single copy gene which share structural features: CWF-localized CWP1 and CWP2 are $\approx 26 \mathrm{kDa}$ with five LRR tandem repeats while CWP3 is $27.3 \mathrm{kDa}$ and has four and a possible fifth LRR tandem repeat. CWP2 is synthesized as a $39 \mathrm{kDa}$-sized precursor that is processed by a cysteine protease releasing the distinctive $13 \mathrm{kDa}$-basic C-terminal extension (TCWP2; Touz et al., 2002; DuBois et al., 2008). All CWPs have a peptide signal sequence at the N-terminus, possibly recognized by a cognate giardial receptor (Svärd et al., 1999) that targets CWP to endoplasmic reticulum (ER), and a cysteine-rich region after the LRR tandem repeats where all 14 cysteine residues are positionally conserved (Mowatt et al., 1995; Luján et al., 1995; Sun et al., 2003). Green fluorescent protein (GFP)-CWP1 chimeras have shown that the N-terminal domain may be needed for sorting CWP to secretory compartments and the LRR tandem repeats may help CWP to associate with CWF material (Hehl et al., 2000). The cysteinerich domain may aid CWP in forming disulfide-bonded heterodimers (Luján et al., 1995; Sun et al., 2003) that are concentrated in the ER lumen potentially promoting ESV biogenesis (Luján \& Touz, 2003). This is supported by the fact that dithiothreitol reduces CWP complexes to monomers, inhibits ESV formation and reversibly converts these secretory granules into ERlike flattened cisternae (Reiner et al., 2001). Likewise the TCWP2 moiety may confer on CWP2 a role as sorting factor for CWP complexes through a likely ligand activity with Giardia-specific ER membrane receptors. This is in turn consistent with the observation that nonencysting trophozoites transfected with haemagglutinin-tagged CWP1-TCWP2 chimera or the whole CWP2 display granules morphologically similar to ESV (Gottig et al., 2006) but these granules are not formed in similarly transfected mammalian cells (Elias et al., 2007). 
CWP are used as markers to define the main phases of encystation: a) "induction", in which encystation stimuli are transduced from surface to nucleus by signaling pathways that activate differential gene expression; (b) "intracellular", involving biosynthesis and trafficking of cyst wall proteins (CWP) and precursors of the GalNAc homopolymer that will form the fibrillar wall matrix; and c) "extracellular", hallmarked by the secretion and extracellular assembly of cyst wall material (Fig. 1).

Several studies have aimed at defining the transport pathway of CW components to the cell surface. Giardia lacks typical Golgi dictyosomes but possesses a basic framework of the vesicular transport system that includes coatomers (COP) I and II (Golgi- and ER-specific, respectively), clathrins, two adaptor protein (AP) complexes and key factors for vesicular targeting and membrane fusion processes such as members of the soluble N-ethylmaleimide-sensitive factor attachment protein receptor (SNARE) and Rab families (Luján \& Touz, 2003; Hehl \& Marti, 2004). Encystation-specific vesicles (ESV) are specialized secretory granules that mature during their traffic from ER to the cell surface that act as the equivalents of late Golgi structures and are also a hallmark of encystation induction. ESV arise from: a) specialized regions of the ER, a trans-Golgi equivalent (Gottig et al., 2006); b) enlarged ER cisternae (Lanfredi-Rangel et al., 2003); or c) homotypic fusion of ER-derived COPII-coated transport vesicles into "pre-Golgi" vesicles (Marti et al., 2003). The modestly increased mRNA expression levels of structural and cargo processing molecules of ESV during encystation induction (Marti et al., 2003) suggest that these structures are derived from structural templates in the ER that differentially recruit components required for sorting and cargo maturation. ESV shape is thought to be changed from irregular to spherical as cargo matura-
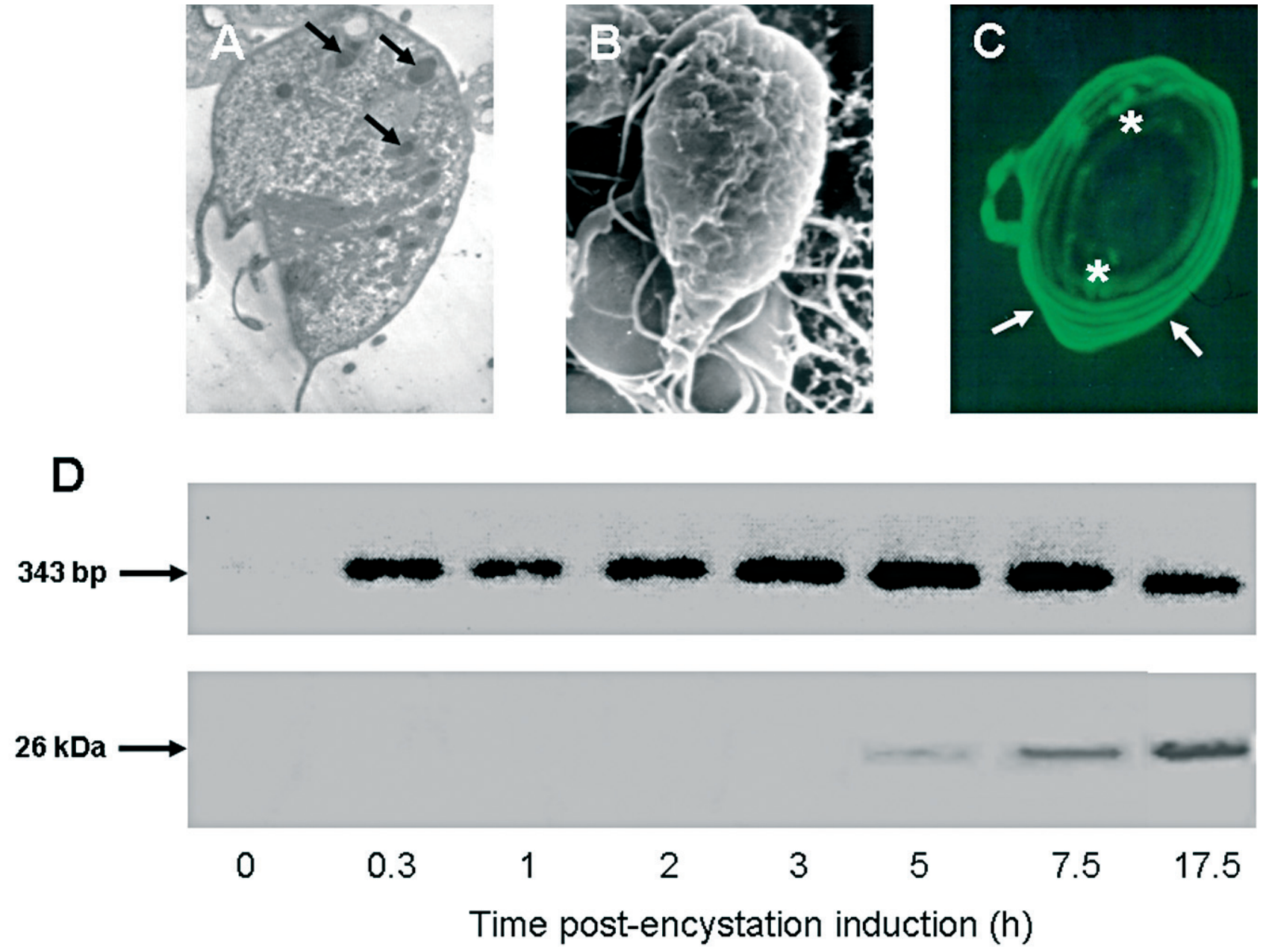

Fig. 1. - Structural and molecular markers of Giardia encystation. A. Transmission electron microscopy of an encysting cell displaying multiple ESVs in the cytoplasm (arrows); B. Scanning electron microscopy of an encysting cell with the cyst wall filamentous material on surface; C. Confocal laser microscopy of an encysting cell stained with the CWP1-specific antibody 5-3C displaying reactivity at ESVs (asterisks) and cell surface (arrows); D. Detection of cwp1 mRNA by semi-quantitative RT-PCR assay (upper panel) and of CWP1 polypeptide by Western blot with monoclonal antibody 5-3C (lower panel). The size of the amplified product and the molecular weight (Mr) of the reactive protein in trophozoites induced to encyst for different time periods are indicated at the left of each panel respectively. 
tion proceeds (Stefanic et al., 2006). This maturation involves three major processes:

- Membrane remodeling

There is an early recruitment of peripheral matrix proteins in membranes of immature ESV including $\beta^{\prime} \mathrm{COP}$, an homolog of yeast GTPase transporter Ypt1p-interacting protein (YiP), Rab11 and dynamin-like protein (DLP), and a late recruitment of others such as the clathrin heavy chain (CLH). Of these, CLH and DLP redistribute mostly to the ESV while the others redistribute to ESV at a lower extent upon encystation progression (Marti et al., 2003).

\section{- Quality control of cargo}

Misfolded cargo molecules are most likely processed by proteasome and Sec61 translocon activities to allow ESV-to-ER retrograde transport of proteins within COPIcoated vesicles where chaperons such as BiP cycle between these compartments (Marti et al., 2003; Stefanic et al., 2006).

- Post-translational modifications of cargo

A critical modification of CWP2 is its processing by a cathepsin B-like cysteine protease (CP2, orf EAA 41050), the most up-regulated of 25 clan CA proteases expressed by Giardia (DuBois et al., 2008), likely releasing the $26 \mathrm{kDa}$ fragment (and hence CWP complexes) to the ESV lumen. CWP2 processing would be also performed by another ESV-contained cathepsin B-like cysteine protease (orf EAA 37074; DuBois et al., 2008) but not so for the peripheral vesicle (PV)-contained cathepsin C-like encystation-specific cysteine protease (ESCP, orf EAA 36907; Touz et al., 2002).

Near to the plasma membrane, ESV appears to carry out two processes: a) fusion with PV (Luján \& Touz, 2003) that acidifies the cargo to limit CP2 activity and/or allow ESCP activity and favor other undefined processing mechanisms preceding the release of $\mathrm{CW}$ material on the cell surface; or b) fission (dispersal) into small secretory vesicles that eventually fuse with the plasma membrane to discharge its content (Hehl \& Marti, 2004). Proposal 'a' is supported by the PV-toESV redistribution of CLH (Marti et al., 2003) while 'b' is consistent with microscope observations of the initial secretion of antigenic [(glyco)polypeptide] CW material as small protrusions on the surface of encysting cells (Erlandsen et al., 1990, 1996). CWP complexes are concentrated, stabilized, sorted and bud in ESV together with chaperons that allow their correct folding (by immunoglobulin heavy chain-binding protein (BiP)) and oligomerization (by protein disulfide isomerases (PDI) 1-3). Another ESV cargo protein (gGSP) helps to maintain low intra-ESV calcium levels to prevent premature assembly and to regulate exocytosis of CWP complexes (Luján \& Touz, 2003).

The release of CW material from ESV is carried out by exocytosis and appears to involve incomplete fusion between plasma and ESV membranes (Benchimol, 2004). The release of ESV contents and CWF formation/assembly may occur stepwise in late encysting cells, i.e., CWP and HCNCp (glyco)polypeptide exposure on the cell surface precedes their co-polymerization with the $[\operatorname{GalNAc}(\beta 1 \rightarrow 3) \operatorname{GalNAc}(\beta 1 \rightarrow 3)]_{\mathrm{n}}$ complex (Argüello-García et al., 2002). This proposal is supported by independent estimations of the time for ESV content release (up to $1 \mathrm{~min}$ ) (Hehl \& Marti, 2004) compared with that required for complete CW assembly (5-6 h.), a process occurring by fibril tip growth (Erlandsen et al., 1996; Argüello-García et al., 2002) and by the presence of CW (glyco) polypeptides in 15-to$100 \mathrm{~nm}$-sized protrusions laid on the surface of encysting cells (Erlandsen et al., 1996) which do not display typical CWF architecture. The cytolocalization of cyst wall synthase (CWS) activity in encysting cells will provide further insights into the process of $\mathrm{CW}$ formation. With respect to the induction phase of encystation, recent experimental and bioinformatics evidence provide new information that allow proposing a model discussed in the sections below.

\section{CHOLESTEROL REGULATION OF GENE TRANSCRIPTION AND GIARDIAL ENCYSTATION}

xtracellular cholesterol promotes giardial growth $\checkmark$ and its depletion triggers encystation (Luján et al. 1996, 1997). This suggests a role for nutrient-cell surface receptor interactions promoting modulation of cell signaling cascades that lead to growth or differentiation. Cholesterol auxotrophy for Giardia growth is unambiguous in spite of the presence of gene transcripts of enzymes from initial steps of the mevalonate pathway. These include hydroxymethylglutaryl CoA synthase (HMGCoA-syn) and the rate-limiting enzyme HMGCoA reductase (HMGCoA-red) (Hernández \& Wasserman, 2006), the de novo synthesis of farnesyl and geranylgeranyl isoprenoids and ubiquinone (Luján et al., 1997) and the presence of sequences with low homology to squalene oxidase, squalene synthetase and lanosterol $14-\alpha$-demethylase in the annotated GiardiaDB. However, the cyclization of squalene to lanosterol and further conversion to cholesterol, demanding at least $11 \mathrm{O}_{2}$ molecules (Ikonen, 2008) are unlikely to occur in Giardia since it is microaerophilic. Instead trophozoites take up cholesterol by calcium-independent endocytosis (Luján et al., 1996, 1997) mediated by a $69-\mathrm{kDa}$ cell surface glycoprotein receptor (CR) recognizing the cholesterol moiety of low density lipoproteins (LDL) (Kaul, 2003) which is antigenically similar in mammalian cells and Giardia (Kaul et al., 2001). In other eukaryotic models, upon interaction 
with cholesterol (i.e., during cell growth) CR displays intrinsic tyrosine kinase activity to generate phosphatidic acid (PA). PA stimulates phospholipase A (PLA $\left.{ }_{2}\right)$ activity in a calcium-dependent manner to produce arachidonic acid (AA) and tromboxans $\left(\mathrm{TxA}_{2}\right)$ increasing intracellular cAMP and cGMP levels, respectively (Kaul, 2003). Protein kinase A (PKA) is activated by cAMP and phosphorylates the precursor form of the $125 \mathrm{kDa}$-sized sterol-responsive element binding protein (pSREBP; Fig. 2) which may be retained in the ER by the SREBP cleavage-activating protein (SCAP) and insulin-induced gene protein (INSIG) (Ikonen, 2008; Kaul, 2003; Yellaturu et al., 2005). In Giardia specific $\mathrm{Ca}^{2+}$-dependent, membrane-associated and alkaline-soluble PLA 2 activities have been demonstrated (Vargas-Villarreal et al., 2007); AA is generated as an intermediate product in the giardial Lands cycle that allows the remodeling of uptaken phospholipids and generation of new ones (Das et al., 2001) and noteworthy endogenous cAMP promotes vegetative growth and the activation phase of excystation (Abel et al., 2001).

In the infected host, trophozoites carried down the colon are differentially exposed to major biliary lipids (bile salts, lecithin and cholesterol) at a slightly alkaline
$\mathrm{pH}$. Encystation is triggered at the lumen of the lower jejunum upon cholesterol depletion by epithelial absorption, bacterial metabolization and a lower cholesterol uptake by trophozoites induced by micellar bile acids (Luján et al., 1997) released at the proximal duodenum and reabsorbed in the lower ileum. CR is not stimulated at this point, CAMP levels decrease and pSREBP is transported within COPII-coated vesicles to the Golgi apparatus and processed by proteolytic cleavage to generate the mature $47 \mathrm{kDa}$-sized form (mSREBP) (Kaul, 2003; Yellaturu et al., 2005) (Fig. 2). In good agreement with these notions cAMP levels in trophozoites are significantly lowered upon deprivation of growth factors (Abel et al., 2001). This partially explains why in vitro encystation can also be achieved using antibodies against CR which may be inactivated catalytically as well as cholesterol endocytosis (Kaul et al., 2001). Likewise infusion of $\mathrm{N}_{2} / \mathrm{CO}_{2}$ would have a similar effect as these gases are able to intercalate within membranes (Alberts et al., 2008) possibly altering CR activity. Moreover, the giardial mSREBP homolog is antigenically and functionally similar to its mammalian counterpart as reported in ovary cells transfected with CWP2 promoter-reporter constructs (Kaul et al., 2001; Worgall et al., 2004).
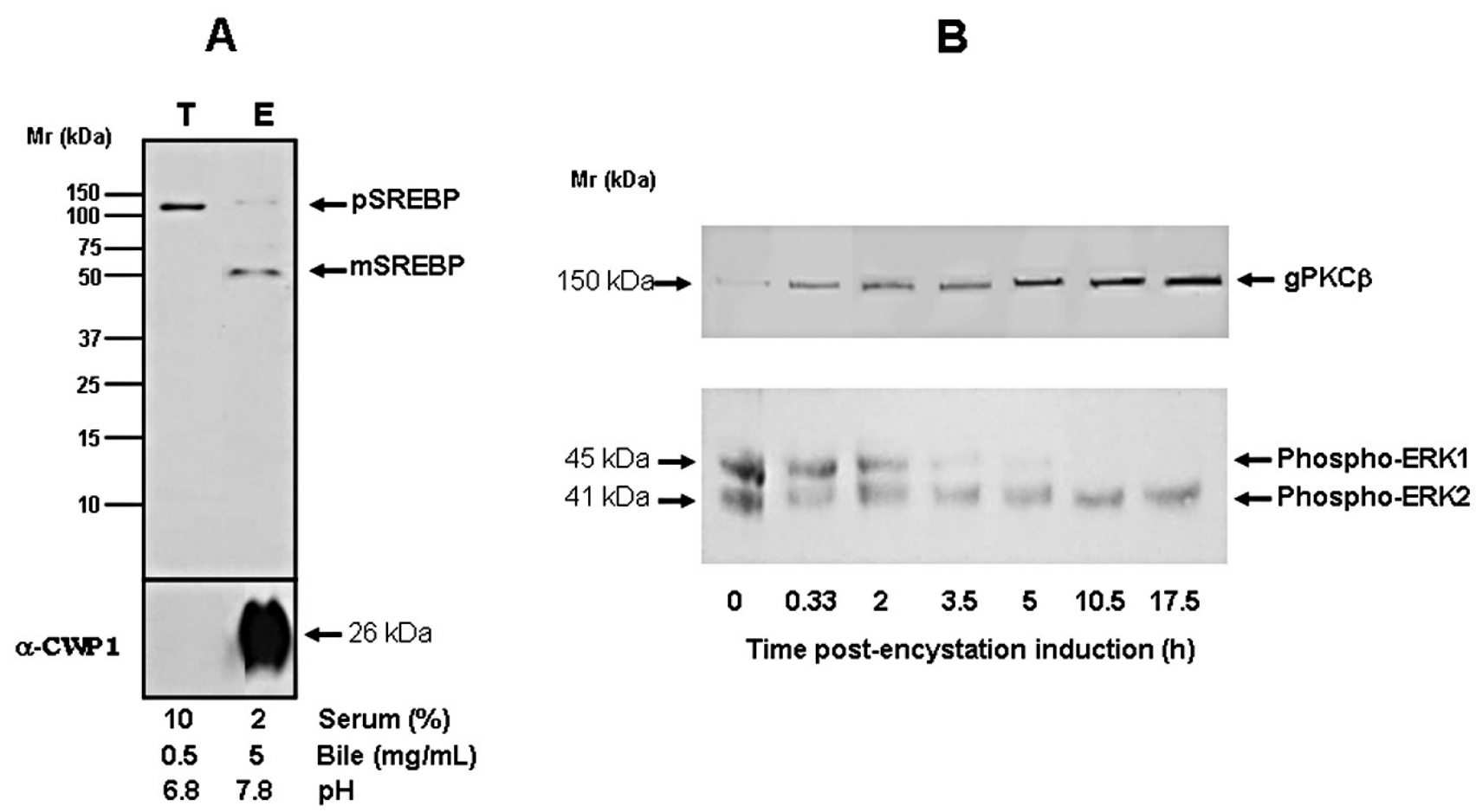

Fig. 2. - A. Western blot of extracts obtained from trophozoites cultured for $36 \mathrm{~h}$ under conditions inducing proliferation (T) and encystation (E) and incubated with monoclonal antibodies against human SREBP-1 (upper panel) and 5-3C antibodies (lower panel). Processing of SREBP from the precursor (pSREBP, $125 \mathrm{kDa}$-sized) to the mature form (mSREBP, $47 \mathrm{kDa}$-sized) is shown. B. Western blot of extracts from trophozoites incubated for different time periods in encystation medium and incubated with antibodies against the catalytic subunit of human PKCBII (upper panel) and with a phospho-ERK antibody generated against a 20-amino acids peptide antigen around the TXY motif of human p44 ERK1. The latter has 65 and $52 \%$ identity with the same region of the giardial ERK1 and 2, respectively (lowerpanel). The levels of ERK1 phosphorylation are markedly decreased after the induction phase of encystation $(3-5 \mathrm{~h})$ has progressed. The relative mobility (Mr) is indicated in $\mathrm{kDa}$ at the left of each panel. 
Transcription factor mSREBP translocates to the nucleus where it interacts with genomic SRE at gene promoters of either the cholesterol biosynthesis pathway ( $H M G C O A-$ syn and HMGCOA-red) and fatty acid biosynthesis ( $L D L r$ ) and CWPs resulting in their up-regulation (Worgall et al., 2004; Ellis et al., 2003) (Fig. 3). However, mSREBP is readily degraded within the mammalian nucleus after a rise in sterol concentration. Of interest, Giardia exhibits increased levels of both HMGCoA-syn and HMGCoA-red transcripts up to $12 \mathrm{~h}$ into encystation progression. These then decline by $24 \mathrm{~h}$ postinduction due to gene down regulation by de novo synthesis of mevalonate-derived metabolites but CWP transcripts remain up-regulated (Hernández \& Wasser-

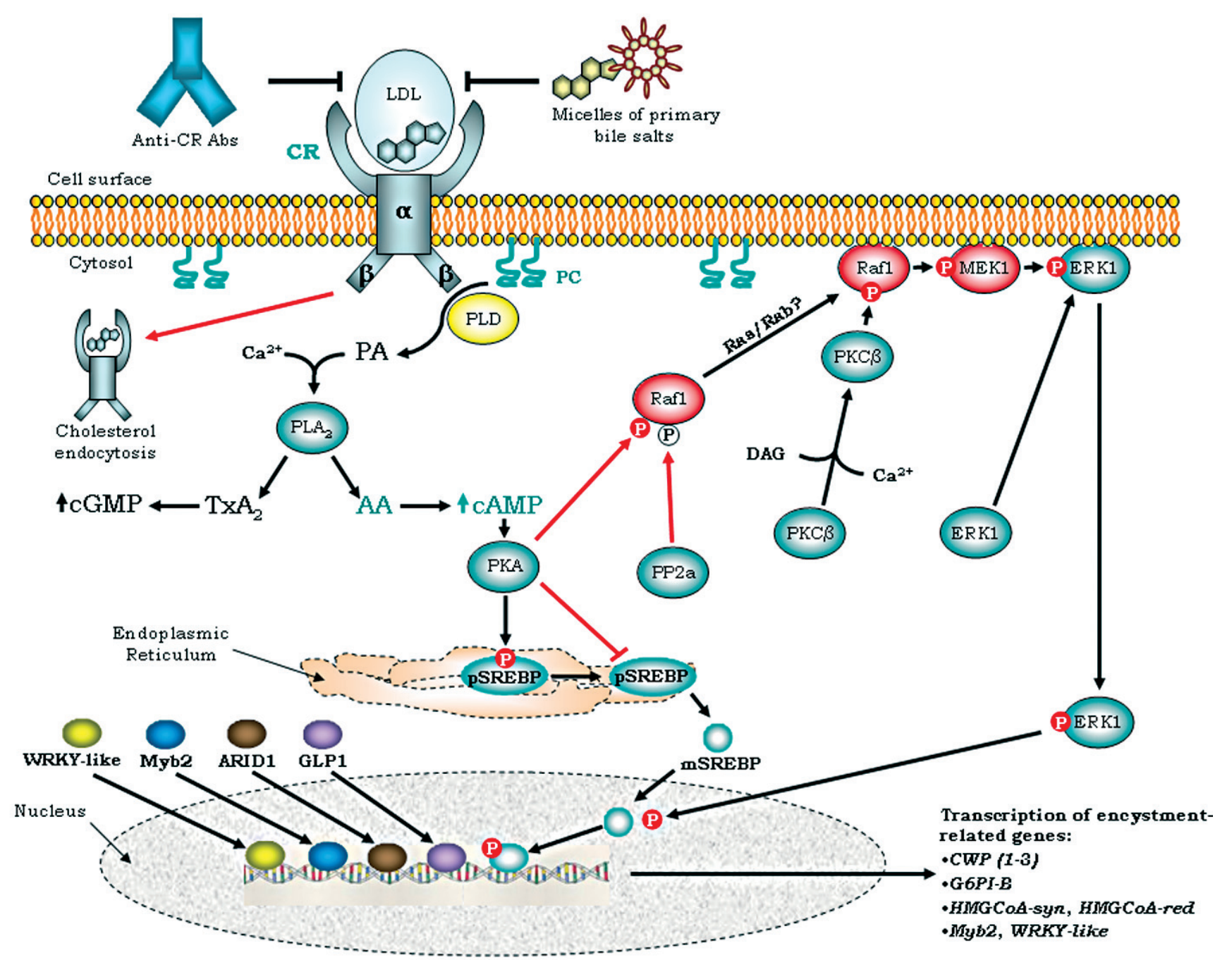

Fig. 3. - Proposed signaling cascades of cholesterol-mediated gene transcription in G. duodenalis and their relation to encystation induction. The giardial CR (that in mammalian cells consists of a $32 \mathrm{kDa}$-sized $\alpha$ subunit and two $19 \mathrm{kDa}$-sized $\beta$ subunits) upon interaction with the cholesterol moiety from LDL may display both cholesterol endocytosis and RTK activity promoting a rise in cAMP levels that is associated with trophozoite motility and replication. PKA activation may keep the $125 \mathrm{kDa}$-sized form (pSREBP) phosphorylated which will be retained at the endoplasmic reticulum. In addition to pSREBP, Raf1 may be phosphorylated by PKA and/or dephosphorylated by PP2a rendering Raf1 in an inactive state. If CR activity is not induced or blocked (e.g., by low levels of extracellular cholesterol or binding by micelles of primary bile salts or CR-specific antibodies) CAMP levels decrease, pSREBP is proteolytically processed to the $47 \mathrm{kDa}$ form (mSREBP) and Raf1 may be activated and translocated to the cell membrane to be phosphorylated by activated PKC $\beta$. Further MEK1 may be phosphorylated by Raf1 and ERK1 re-localizes to the membrane and after phosphorylation by MEK1 migrates to the nucleus where mSREBP is activated. This molecule together with other transcription factors (GLP1, ARID1, Myb2 and WRKY-like) may interact with promoters activating transcription of genes encoding encystation-related markers and enzymes of the mevalonate pathway. Green ovals represent signaling molecules experimentally identified and red ovals are those deduced by bioinformatics data from the GiardiaDB (see Table I for details).

Abbreviations: AA: arachidonic acid; CR: cholesterol receptor; ERK1: extracellular signal-activated kinase 1; HMGCoA syn/red: hydroxymethylglutaryl coenzyme A synthase/reductase; LDL: low density lipoproteins; PA: phosphatidic acid; PKA/C: protein kinase A/C; PLA 2 /D: phospholipase $\mathrm{A}_{2} / \mathrm{D}$; p/mSREBP: precursor/mature sterol-response element binding protein; PP2a: protein phosphatase 2a; Raf1: mitogenactivated protein kinase $1 ; \mathrm{TxA}_{2}$ : thromboxan $\mathrm{A}_{2}$. 
man, 2006). Extracellular cholesterol is indeed a negative regulator of CWP transcription (Luján et al., 1997) and the SREBP pathway in Giardia (Worgall et al., 2004) ultimately blocking encystation.

\section{ARE BILE SALTS RELEVANT FOR ENCYSTATION INDUCTION?}

工 $\mathrm{t}$ $\mathrm{t}$ has been proposed that bile salt micelles incorporate ionized fatty acids at slightly alkaline $\mathrm{pH}$ reducing their giardial cytotoxicity (Gillin et al., 1989) and that these micelles stimulate giardial encystation by an indirect, inhibitory effect on cholesterol uptake by trophozoites (Luján et al., 1997). Nevertheless, bile or bile acid deprivation at the duodenum of infected CF-1 mice by surgical cholestasis or by diets containing a bile acid-sequester resin (cholestyramine) dramatically reduced Giardia cyst shedding without affecting trophozoite establishment and replication (Erlandsen, 2005). This strongly supports the view that high bile concentrations and a slightly alkaline $\mathrm{pH}$ in TYI-S-33 medium efficiently mimics conditions found in the small intestine environment (Gillin et al., 1987) and that bile acids are a "natural stimulus" for giardial encystation. Moreover glycine and taurine $\mathrm{N}$-acyl conjugates of primary bile salts (cholate and chenodeoxycholate) are far more efficient at inducing Giardia encystation than secondary salts (deoxycholate) (Gillin et al., 1987). Indeed taurodeoxycholate at micellar concentrations $(\geq 5 \mathrm{mM}$ ) inhibited the transfer of cholesterol from medium to trophozoites (Luján et al., 1997) although this secondary bile salt stimulated encystation with very low efficiency (Gillin et al., 1987). Inhibition of cholesterol uptake by bile salts is an event not sufficient in itself to complete the encystation process. Thus an interaction between chenodeoxycholate-rich micelles and cell surface components is likely to occur in agreement with the known selectivity of CR for the cholesterol moiety of LDLs taking into account that primary bile acids are the closest resembling compounds to cholesterol while secondary bile acids are derived from primary ones by bacterial dehydroxylation (Alberts et al., 2008). Further, the chenodeoxycholate moiety is more similar to cholesterol than cholate and regardless of whether chenodeoxycholate is conjugated to glycine or taurine, the cyst yields are comparable to those obtained with glycocholate (see Fig. 3 of Gillin et al., 1987). However the less voluminous glycine group might have a lesser hindrance effect than the more voluminous taurine group favoring CR inactivation as glycocholate is more effective than taurocholate inducing encystation (Gillin et al., 1987).

\section{CELL SIGNALING IN GIARDIAL ENCYSTATION: EXPERIMENTAL AND BIOINFORMATICS INSIGHTS}

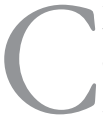
holesterol-mediated regulation of gene transcription is associated with encystation induction in Giardia and mSREBP production appears to be a critical step for biosynthesis and accumulation of CWPs. However, a signaling cascade is needed to upregulate SRE-containing gene promoters by mSREBP. The transcriptional activity of MSREBP is decreased by its ubiquitylation and/or sumoylation while the effect of its phosphorylation depends on the kinase involved: extracellular-signal regulated kinase (ERK)-mediated phosphorylation increases transcription by mSREBP while it decreases by PKA or glycogen synthase kinase (GSK)mediated phosphorylation (Arito et al., 2008). Giardial homologues of the mitogen-activated protein kinases (MAPKs) ERK1 and ERK2 have been identified (Ellis et al., 2003). Endogenous ERK1 exhibited increased activity, slightly higher phosphorylation and a noticeable partial relocalization to the cell membrane at an early time post-encystation induction $(\approx 2 \mathrm{hrs}$; see Figs. 6A and 6B of Ellis et al., 2003) while ERK2 at this stage relocates from the membrane to the cytosol concomitant with a decrease in its activity (Ellis et al., 2003). Interestingly, Giardia and Dictyostelium discoideum ERK2s are closely related at the amino acid (aa) level (66\% identity and $84 \%$ homology, expectation value of 8e-130) (Table I) with a co-localized cAMPdependent PKA phosphorylation site (KRiT) that in the slime mold is involved in activation and translocation of ERK2 to the nucleus during the multicellular development response to extracellular factors that increase intracellular cAMP levels. These observations suggest the involvement of ERK1 in giardial mSREBP phosphorylation.

Considering MAPK activation by the dual specificity MAPK kinases 1 and 2 (MAPKKs = MEK1/2; McCubrey et al., 2007), there is one giardial homolog of MEK1 (STE20 type) in the GiardiaDB (orf 22165) (Table I). The sequences of human (393 aa long) and giardial (357 aalong) MEK1s show at most $39 \%$ identity and $56 \%$ homology (e.v. 1.8e-40) over a 209 aa-stretch. Giardia MEK1 displays the ATP-binding and serine/threonine signature domains of bona fide MEK1s. Likewise one homolog to MAPKKK1 (syn. Raf1, MEK kinase 1), a MEK-phosphorylating small GTPase, is present in the annotated GiardiaDB (orf 15868) (Table I). Its aa sequence displays at most $26 \%$ identity and $46 \%$ homology to MEK kinase 1 from Gallus gallus with an e.v. 9e-17 over a 206 aa-stretch. The giardial homolog exhibits the two zinc Finger profile domains (SWIN and RING) near the N-terminus present in Raf-related 


\begin{tabular}{|c|c|c|c|c|}
\hline $\begin{array}{l}\text { Signalling } \\
\text { protein }\end{array}$ & $\begin{array}{c}\text { Orf in GiardiaDB } \\
\text { (http://www.giardiadb.org/ } \\
\text { giardiadb/) }\end{array}$ & $\begin{array}{c}\text { GenBank }^{\mathrm{a}} / \mathrm{NCB1}^{\mathrm{b}} \\
\text { Accession No. }\end{array}$ & $\begin{array}{l}\text { Maximal identity value } \\
\text { and homolog species } \\
\text { (full length/partial) }\end{array}$ & Reference \\
\hline ERK1 & GL50803_17563 & AAN73429.1* & $51 \%$, Dictyostelium discoideum (fl) & Ellis et al. (2003) \\
\hline ERK2 & GL50803_22850 & AAN73430.1* & $66 \%$, D. discoideum $(\mathrm{fl})$ & Ellis et al. (2003) \\
\hline MEK1-like & GL50803_22165 & XP_001704049.1 & $39 \%$, Arabidopsis thaliana $(\mathrm{p})$ & This review \\
\hline RAF1-like & GL50803_15868 & XP_001705158.1 & $26 \%$, Gallus gallus $(\mathrm{p})$ & This review \\
\hline cPKC $\beta$-like & GL50803_86444 & XP_001704119.1b & $38 \%$, Danio rerio $\mathrm{PKC} \beta \mathrm{I}(\mathrm{p})$ & Bazán-Tejeda et al. (2007) \\
\hline rPKC $\beta$-like & GL50803_137754 & EDO76444.1* & $\begin{array}{l}36 \% \text {, Tribolium castaneum } \\
\text { AGAP012366-PA-like }(\mathrm{p})\end{array}$ & Bazán-Tejeda et al. (2007) \\
\hline cPKA & GL50803_11214 & AAG09429.1* & $48 \%, D$. discoideum $(\mathrm{fl})$ & Abel et al. (2001) \\
\hline rPKA & GL50803_9117 & AAV71055.1* & $37 \%$, D. discoideum $(\mathrm{fl})$ & Gibson et al. (2006) \\
\hline cPP2A & GL50803_5010 & $\mathrm{XP} \_767901^{\mathrm{b}}$ & $63 \%$, Homo sapiens $(\mathrm{fl})$ & Lauwaet et al. (2007) \\
\hline
\end{tabular}

Table I. - Molecules proposed in the MAPK/ERK signalling pathway leading to SREBP activation during induction of G. duodenalis encystation. (Fig. 3). Codes: Orf: open reading frame; c: catalytic subunit; r: regulatory subunit.

kinases but the kinase domain at the C-terminus in Raf prototypes (e.g. human v-Raf-1, 648 aa-long) is atypical or truncated since the putative giardial homolog is shorter than the Raf-related G. gallus sequence displaying this domain (606 vs 1346 aa, respectively).

In addition Raf-related sequences, including giardial Raf1-like, lack a Ras-binding domain (RBD) that allows Ras-mediated Raf translocation to cell membrane (McCubrey et al., 2007). To date one putative RBD has been described in the phosphatidylinositol-3-kinase-1 (PI3K1) sequence of this protist (Cox et al., 2006) and a Ras-like GTPase sequence was reported in the annotated GiardiaDB (orf 9718) suggesting that Ras-like is preferentially involved in the partially characterized PI3K signaling pathway of G. duodenalis (Cox et al., 2006; Morrison et al., 2002). Therefore giardial Raf1like may be recruited to the cell membrane during encystation by another small GTPase such as a Rab protein, of which at least nine members are present in the GiardiaDB. Together the bioinformatics evidence suggests a pivotal role for the Raf1/MEK1/ERK1 pathway in the induction phase of giardial encystation (Fig. 3). As an upstream activator of this pathway, Raf1 activity is positively regulated by protein kinase $\mathrm{C}$ and negatively regulated by PKA and protein phosphatase $2 \mathrm{~A}$ (PP2A) (McCubrey et al., 2007). The catalytic subunit of giardial PKA (PKAc) retains the ability of protein expression at $2 \mathrm{~h}$ post-encystation induction without relocalizing from cytoskeletal structures to the cell membrane (Gibson et al., 2006). In the case of giardial PP2A, it has been observed that the catalytic subunit (PP2A-C) is associated to basal bodies/centrosomes, ventral disk and paraflagellar dense rods (PDRs) in trophozoites. Shortly after encystation induction (up to $4 \mathrm{~h}$ ) it was no longer found in the anterior PDRs, its mRNA and protein levels decreased, no redistribution ocurred and it was localized only in the CW of excysting cells. Moreover, trophozoites transfected to stably express antisense PP2A-C mRNA showed decreased levels of PP2A-C and CWP1 proteins, less ESV formation and lower encystation (Lauwaet et al., 2007). Based on a similar localization of PKAc, ERK1, centrin, calmodulin and PP2A-C in trophozoites and changes of their cytolocalization upon encystation/excystation induction, it was proposed that PP2A-C acts as a dual regulator: initially for pathways leading to loss of adhesion, motility and cell division during ongoing encystation and then in pathways leading to reactivation of these functions during excystation (Lauwaet et al., 2007). This conclusion supports the absence of interactions between PP2A and Raf1-like at trophozoite membrane during encystation induction. On the other hand, protein kinase $\mathrm{C}$ (PKC) homologs including one conventional ( $\beta$ II-like), three novel $(\delta, \varepsilon, \theta)$ and one atypical

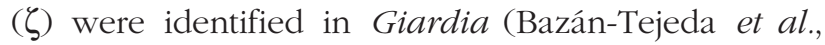
2007). Of these, endogenous PKC $\beta I I-l i k e$ required $\mathrm{Ca}^{2+}$, phosphatidylserine and diolein/diacylglycerol for full kinase activity; this protein was redistributed from cytoplasm to cell membrane during the first 10-30 min after encystation induction and by $2 \mathrm{~h}$ post-encystation induction this kinase relocated again to the cytoplasm. General or $\beta$-specific PKC inhibitors affected encystation but not cell growth (Bazán-Tejeda et al., 2007). These data suggest the activation of $P K C \beta$ as it occurs for ERK1 but at an earlier time post-encystation induction (10-30 min. vs $2 \mathrm{~h}$. respectively) as expected from the signaling cascade (Raf1/MEK1/ERK1) proposed to be involved in this process (Figs 2 and 3). As per other models, PKC activity remains inhibited when the $\mathrm{CR}$ is stimulated to down-regulate the expression of some transcription factors such as c-myc by mechanisms that may inhibit the accumulation of PKC cofactors as diacylglycerol (Sikand et al., 2006). This observation together with possible SREBP maturation when the CR-dependent cAMP/PKA pathway is switched off suggest the existence of regulation points at the interphase of the CR-dependent and the MAPK-dependent pathways in this protist.

As far as the signaling regulation of growth and differentiation in Giardia is concerned, encystation commit- 
ment has to be achieved at the expense of cell growth in which the transducing activity of some receptors for growth factors or nutrients localized on the cell surface is crucial. Most of these receptors have intrinsic tyrosine kinase activity and are included in the so called receptor tyrosine kinases (RTKs) family. In spite that no tyrosine-specific kinases have been predicted from the GiardiaDB (Morrison et al., 2007) protein tyrosine phosphorylation has been shown present in this parasite (Parsons et al., 1993; Luján et al., 1994). Besides CR, Giardia has at least a RTK for insulin-like growth factor (ILGFR) that promotes its growth and cysteine uptake (Luján et al., 1994). These ILGFRs are linked to the Ras/PI3K/PTEN/Akt/TOR pathway (Alberts et al., 2008) which has been partially characterized and proposed to be functional in this protist on the basis of transcriptional analyses and bioinformatics data mined from GiardiaDB and related sources (Morrison et al., 2002; Cox et al., 2006; Hernández et al., 2007).

In addition a significant inhibitory effect of PI3K inhibitors on trophozoite growth was observed (Cox et al., 2006; Hernández et al., 2007) strongly arguing against its involvement in differentiation induction whenever giardial Akt (or protein kinase B) was reported as upregulated at the transcriptional level by $17 \mathrm{~h}$ postinduction of encystation (Kim et al., 2005), a time much longer than that proposed herein for the induction phase $(3-5 \mathrm{~h})$. In this context other signal-recruiting molecules such as the protein 14-3-3 identified in G. duodenalis exhibits intracellular changes of localization during encystation (from cytosolic to partially intranuclear) by $12 \mathrm{~h}$ post-encystation induction, a time at which ESVs have already been formed (Lalle et al., 2006). These latter observations lead to hypothesize that other signaling pathways involving mediators as PKB, PI3K2 and protein 14-3-3 have a role in encystation but at later stages than the induction one.

\section{SREBP, ANOTHER FACTOR IN THE SCENARIO}

E arly encystation markers including CWPs 1-3 and G6PI-B have similar biosynthetic kinetics (Luján et al., 1997; Steimle et al., 1997; Mowatt et al., 1995; Sun et al., 2003) suggesting that their expression is regulated by similar signaling pathways although other transcriptional factors are also involved. In spite of lacking 8 of 12 general eukaryotic transcription factors (Best et al., 2004), Giardia possesses at least four other transcription factors that are overexpressed in encystation and bind to and transactivate gene promoters of encystation markers: GARP-like protein 1 (GLP1) and AT-rich interaction domain pro- tein 1 (ARID1) for cwp1 promoter; Myb2 for promoters of cwp 1-3, g6pi-b and myb2 itself and a WRKY protein homolog for promoters of cwp 1-2, myb2 and wrky itself (Sun et al., 2002; Sun et al., 2006; Wang et al., 2007; Pan et al., 2009). The manipulated overexpression of some of these transcription factors provided further insights: for instance, the constitutive over-expression of Myb2 moderately increased cwp1 expression $(\approx 3.7$-fold $)$ as compared with levels observed during bile-induced encystation $(\approx 47$-fold; Huang et al., 2008). This suggests that all these transcription factors interact to reach expression levels beyond a threshold to allow encysting cells to progress to ESV biogenesis and biosynthesis of precursors of the $[\text { GalNAc }(\beta 1 \rightarrow 3) \text { GalNAc }(\beta 1 \rightarrow 3)]_{n}$ polymer. Of note, when ERK1 was constitutively over-expressed, transfected trophozoites showed increased levels of cwp 1-2, wrky and myb2 mRNAs (Pan et al., 2009), which provides additional evidence that these encystation-regulated markers may be downstream elements of the MAPK/ ERK pathway described herein (Fig. 3).

To date, there is some evidence of involvement of the MAPK/ERK signaling pathway in processing and regulation of SREBP activity, while for GLP1, ARID1, Myb2 and WRKY-like factors the accumulated evidence mostly relies on characterization of their DNA-binding capacities and regulation of cwp expression.

\section{CONCLUSION}

$\circlearrowleft$ iardial encystation is a multifactorial process where cholesterol starvation or bile acid micelles are necessary to accomplish the induction phase but not sufficient to complete cyst biogenesis. This phase includes transcription regulation of genes encoding early encystation markers (CWPs 1-3 and G6PI-B) by a cholesterol-mediated process which requires inactivation of the $\mathrm{CR}$-coupled signaling that may be accomplished by primary bile acid-micelles and even by gas infusion or antibody binding. Experimental and bioinformatics data suggest that this inactivation is concomitant with the activation of a Raf1/MEK1/ ERK1 pathway leading to SREBP maturation and activation. It is necessary to define whether the other well characterized encystation-induced transcription factors are regulated by similar or distinct signaling pathways. Characterizing these processes at a functional level will reveal new targets for diagnosis, drug design and prophylactic intervention similarly to the cyst wall polysaccharide-targeting drugs and nucleotide-based cyst wall biosynthesis inhibitors (Jarroll \& Sener, 2003; Suk et al., 2007) and the engineered expression of CWP 2 in bacterial vectors for transmission-blocking vaccines (Lee \& Faubert, 2006; Abdul-Wahid \& Faubert, 2007). 


\section{ACKNOWLEDGEMENTS}

The authors are grateful to Jacqui Upcroft and Edward Jarroll for critically reviewing the manuscript. This work was supported by ConacytMexico grant No. 49724 and ECOS-ANUIES grant No. MO6SO3 France-Mexico.

\section{REFERENCES}

Abdul-Wahid A. \& Faubert G. Mucosal delivery of a transmission-blocking DNA vaccine encoding Giardia lamblia CWP2 by Salmonella typhimurium bactofection vehicle. Vaccine, 2007, 25, 8372-8383.

Abel E.S., Davids B.J., Robles L.D., Loflin C.E., Gillin F.D. \& Chakrabarti R. Possible roles of protein kinase A in cell motility and excystation of the early diverging eukaryote Giardia lamblia. J. Biol. Chem., 2001, 276, 10320-10329.

Alberts B., Johnson A., Lewis J., RafF M., Roberts K., Walter P., Wilson J. \& Hunt T. (eds), Molecular Biology of the Cell, Garland Science Inc., 2008, $5^{\text {th }}$ edition.

Argüello-Garcia R., Argüello-López C., González-Robles A., Castillo Figueroa A.M. \& Ortega-Pierres M.G. Sequential exposure and assembly of cyst wall filaments on the surface of encysting Giardia duodenalis. Parasitology, 2002, $125,209-219$.

Arito M., Horiba T., Hachimura S., Inoue J. \& Sato R. Growth factor-induced phosphorylation of sterol regulatory element-binding proteins inhibits sumoylation, thereby stimulating the expression of their target genes, low density lipoprotein uptake, and lipid synthesis. J. Biol. Chem., 2008, 283, 15224-15231.

BazÁn-Tejeda M.L., Argüello-Garcia R., Bermúdez-Cruz R.M., Robles-Flores M \& Ortega-Pierres G. Protein kinase C isoforms from Giardia duodenalis: identification and functional characterization of a beta-like molecule during encystment. Arch. Microbiol., 2007, 187, 55-66.

Benchimol M. The release of secretory vesicle in encysting Giardia lamblia. FEMS Microbiol Lett., 2004, 235, 81-87.

Best A.A., Morrison H.G., McArthur A.G., Sogin M.L. \& Olsen G.J. Evolution of eukaryotic transcription: insights from the genome of Giardia lamblia. Genome Res, 2004, 14, 15371547.

Cox S.S.E., van der Giezen M., Tarr S.J., Crompton M.R. \& TOVAR J. Evidence from bioinformatics, expression and inhibition studies of phosphoinositide-3 kinase signalling in Giardia intestinalis. BMC Microbiol., 2006, 6, 45.

Davids B.J., Reiner D.S., Birkeland S.R., Preheim S.P., Cipriano M.J., McArthur A.G. \& Gillin F.D. A new family of giardial cysteine-rich non-VSP protein genes and a novel cyst protein. PLOS ONE, 2006, 1, e44.

Das S., Castillo C. \& Stevens T. Phospholipid remodeling/ generation in Giardia: the role of the Lands cycle. Trends Parasitol., 2001, 17, 316-319.

DuBois K.N., Abodeely M., Sakanari J., Craik C.S., Lee M., McKerRow J.H. \& SAJID M. Identification of the major cysteine protease of Giardia and its role in encystation. J. Biol. Chem., 2008, 283, 18024-18031.

Eluis J.G., Davila M. \& Chakrabarti R. Potential involvement of extracellular signal-regulated kinase 1 and 2 in encystation of a primitive eukaryote, Giardia lamblia. Stage-specific activation and intracellular localization. J. Biol. Chem., 2003, 278, 1936-1945.

Erlandsen S.L., Macechko P.T., van Keulen H. \& Jarroll E.L. Formation of the Giardia cyst wall: studies on extracellular assembly using immunogold labeling and high resolution field emission SEM. J. Eukaryot. Microbiol., 1996, 43, 416-429.

ERLANDSEN S. Reduction in fecal excretion of Giardia cysts: effect of cholestasis and diet. J. Parasitol., 2005, 91, 14821484 .

Gibson C., Schanen B., Chakrabarti D. \& Chakrabarti R. Functional characterisation of the regulatory subunit of cyclic AMP-dependent protein kinase A homologue of Giardia lamblia: Differential expression of the regulatory and catalytic subunits during encystation. Int. J. Parasitol., 2006, 36, 791-799.

Giluin F.D., Reiner D.S., Gault M.J., Douglas H., Das S., WunDERLICH A. \& SAUCH J.F. Encystation and expression of cyst antigens by Giardia lamblia in vitro. Science, 1987, 235, 1040-1043.

Gillin F.D., Boucher S.E., Rossi S.S. \& ReIner D.S. Giardia lamblia: the roles of bile, lactic acid, and $\mathrm{pH}$ in the completion of the life cycle in vitro. Exp. Parasitol., 1989, 69, 161-174.

Gottig N., Elías E.V., Quiroga R., Nores M.J., Solari A.J., Touz M.C. \& Luján H.D. Active and passive mechanisms drive secretory granule biogenesis during differentiation of the intestinal parasite Giardia lamblia. J. Biol. Chem., 2006, 281, 18156-18166.

Hehl A.B. \& MARTI M. Secretory protein trafficking in Giardia intestinalis. Mol. Microbiol., 2004, 53, 19-28.

Hernández P.C. \& Wasserman M. Do genes from the cholesterol synthesis pathway exist and express in Giardia intestinalis? Parasitol. Res., 2006, 98, 194-199.

Hernández Y., Zamora G., Ray S., Chapoy J., Chavez E., Valvarde R., Williams E., Aley S.B. \& Das S. Transcriptional analysis of three major putative phosphatidylinositol kinase genes in a parasitic protozoan, Giardia lamblia. J. Eukaryot. Microbiol., 2007, 54, 29-32.

Huang Y.C., Su L.H., Lee G.A., Chiu P.W., Cho C.C., Wu J.Y. \& Sun C.H. Regulation of cyst wall protein promoters by Myb2 in Giardia lamblia. J. Biol. Chem., 2008, 283, 3102131029.

IKONEN E. Cellular cholesterol trafficking and compartmentalization. Nat. Rev. Mol. Cell. Biol., 2008, 9, 125-138.

Jarroll E.L., MacechKo P.T., Steimle P.A., Bulik D., Karr C.D., van Keulen H., Paget T.A., Gerwig G., Kamerling J., VlieGENTHART J. \& ERLANDSEN S. Regulation of carbohydrate metabolism during Giardia encystment. J. Eukaryot. Microbiol., 2001, 48, 22-26.

Jarroll E.L. \& Sener K. Potential drug targets in cyst-wall biosynthesis by intestinal protozoa. Drug Resist. Updat., 2003, 6, 239-246. 
Jiménez-Garcia L.F., Zavala G., Chávez-Munguía B., RamosGodinez M.P., López-Velazquez G., Segura-Valdez M.L., Montañez C., Hehl A.B., Argüello-García R. \& OrtegaPIERRES G. Identification of nucleoli in the early branching protist Giardia duodenalis. Int. J. Parasitol., 2008, 38, 12971304.

KaUl D., RAni R. \& SEHgal R. Receptor-Ck regulates Giardia encystation process. Mol. Cell. Biochem., 2001, 225, 167169.

KAUL D. Cholesterol-receptor-mediated genomics in health and disease. Trends Mol. Med., 2003, 9, 442-449.

KARR C.D. \& JARROLL E.L. Cyst wall synthase: N-acetyl galactosaminyltransferase activity is induced to form the novel $\mathrm{N}$-acetylgalactosamine polysaccharide in the Giardia cyst wall. Microbiology, 2004, 150, 1237-1243.

Keeling P.J. \& Brugerolle G. Evidence from SSU rRNA phylogeny that Octomitus is a sister lineage to Giardia. Protist., 2006, 157, 205-212.

KIM K.T., Mok M.T. \& Edwards M.R. Protein kinase B from Giardia intestinalis.Biochem. Biophys. Res. Commun., 2005, 334, 333-341.

Lalle M., Salzano A.M., Crescenzi M. \& Pozio E. The Giardia duodenalis $14-3-3$ protein is post-translationally modified by phosphorylation and polyglycylation of the C-terminal tail. J. Biol. Chem., 2006, 281, 5137-5148.

Lanfredi-Rangel A., Attias M., Reiner D.S., Gillin F.D. \& DE SouzA W. Fine structure of the biogenesis of Giardia lamblia encystation secretory vesicles. J. Struct. Biol., 2003, 143, 153-163.

Lauwaet T., Davids B.J., Torres-Escobar A., Birkeland S.R., Cipriano M.J., Preheim S.P., Palm D., Svärd S.G., McArthur A.G. \& Gilurn F.D. Protein phosphatase 2A plays a crucial role in Giardia lamblia differentiation. Mol. Biochem. Parasitol., 2007, 152, 80-89.

LeE P. \& Faubert G.M. Expression of the Giardia lamblia cyst wall protein 2 in Lactococcus lactis. Microbiology, 2006, 152, 1981-1990.

LujÁN H.D., MowatT M.R. \& NASH T.E. Lipid requirements and lipid uptake by Giardia lamblia trophozoites in culture. J. Eukaryot. Microbiol., 1996, 43, 237-242.

LujÁN H.D., MowatT M.R. \& NASH T.E. Mechanisms of Giardia lamblia differentiation into cysts. Microbiol. Mol. Biol. Rev., 1997, 61, 294-304.

LujÁN H.D. \& Touz M.C. Protein trafficking in Giardia lamblia. Cell. Microbiol., 2003, 5, 427-434.

Marti M. \& Hehl A.B. Encystation-specific vesicles in Giardia: a primordial Golgi or just another secretory compartment? Trends Parasitol., 2003, 19, 440-446.

McCubrey J.A., Steelman L.S., Chappell W.H., Abrams S.L., Wong E.W., Chang F., Lehmann B., Terrian D.M., Milella M., Tafuri A., Stivala F., Libra M., Basecke J., Evangelisti C., Martelli A.M. \& Franklin RA. Roles of the Raf/MEK/ERK pathway in cell growth, malignant transformation and drug resistance. Biochim. Biophys. Acta, 2007, 1773, 12631284 .

Morrison H.G., Zamora G., Campbell R.K. \& Sogin M.L. Inferring protein function from genomic sequence: Giardia lamblia expresses a phosphatidylinositol kinase-related kinase similar to yeast and mammalian TOR. Comp. Biochem. Physiol. B Biochem. Mol. Biol., 2002, 133, 477-491.

Morrison H.G., McArthur A.G., Gillin F.D., Aley S.B., Adam R.D., Olsen G.J., Best A.A., Cande W.Z., Chen F., Cipriano M.J., Davids B.J., Dawson S.C., Elmendorf H.G., Hehl A.B., Holder M.E., Huse S.M., Kim U.U., LaseK-Nesselquist E., Manning G., Nigam A., Nixon J.E., Palm D., Passamaneck N.E., Prabhu A., Reich C.I., Reiner D.S., Samuelson J., Svärd S.G. \& Sogin M.L. Genomic minimalism in the early diverging intestinal parasite Giardia lamblia. Science, 2007, 317, 1921-1926.

Mowatt M.R., Luján H.D., Cotten D.B., Bowers B., Yee J., NASH T.E. \& STiBBS H.H. Developmentally regulated expression of a Giardia lamblia cyst wall protein gene. Mol. Microbiol., 1995, 15, 955-63.

Parsons M., Valentine M. \& Carter V. Protein kinases in divergent eukaryotes: Identification of protein kinase activities regulated during trypanosome development. Proc. Natl. Acad. Sci., 1993, 90, 2656-2660.

Pan Y.J., Cho C.C., KaO Y.Y. \& Sun C.H. A novel WRKY-like protein involved in transcriptional activation of cyst wall protein genes in Giardia lamblia. J. Biol. Chem., 2009 (in press).

Sikand K., Kaul D. \& Varma N. Receptor Ck-dependent signaling regulates hTERT gene transcription. BMC Cell. Biol., 2006, 7, 2.

Stefanic S., Palm D., Svärd S.G. \& Hehl A.B. Organelle proteomics reveals cargo maturation mechanisms associated with Golgi-like encystation vesicles in the early-diverged protozoan Giardia lamblia. J. Biol. Chem., 2006, 281, 7595-7604.

Steimle P.A., Lindmark D.G. \& Jarroll E.L. Purification and characterization of encystment-induced glucosamine 6phosphate isomerase in Giardia. Mol. Biochem. Parasitol., 1997, 84, 149-153.

Sterling C.R., Kutob R.M., Gizinski M.J., Verastegui M. \& SteTZENBACH L. Giardia detection using monoclonal antibodies recognizing determinants of in vitro derived cysts, in: Advances in Giardia Research. Wallis P.M. \& Hammond B.R. (eds), University of Calgary Press, 1988, 219-222.

Sun C.H., Palm D., McArthur A.G., Svärd S.G. \& Gillin F.D. A novel Myb-related protein involved in transcriptional activation of encystation genes in Giardia lamblia. Mol. Microbiol, 2002, 46, 971-984.

Sun C.H., McCaffery J.M., Reiner D.S. \& Gildin F.D. Mining the Giardia lamblia genome for new cyst wall proteins. J. Biol. Chem., 2003, 278, 21701-21708.

Sun C.H., Su L.H. \& GILLIN F.D. Novel plant-GARP-like transcription factors in Giardia lamblia. Mol. Biochem. Parasitol., 2006, 146, 45-57.

Suk D.H., Rejman D., Dykstra C.C., Pohl R., Pankiewicz K.W. \& Patterson S.E. Phosphonoxins: rational design and discovery of a potent nucleotide anti-Giardia agent. Bioorg. Med. Chem. Lett., 2007, 17, 2811-2816.

SVÄrd S.G, RAFFERTy C., MCCAFFERY J.M., SMith M.W., ReINER D.S. \& Gillin F.D. A signal recognition particle receptor gene from the early-diverging eukaryote, Giardia lamblia. Mol. Biochem. Parasitol., 1999, 98, 253-264. 
Tovar J., León-Avila G., Sánchez L.B., Sutak R., Tachezy J., van der Giezen M., Hernández M., Müller M. \& LucocQ JM. Mitochondrial remnant organelles of Giardia function in iron-sulphur protein maturation. Nature, 2003, 426, 172176.

Vargas-Villarreal J., Escobedo-Guajardo B.L., Mata-Cárdenas B.D., Palacios-Corona R., Cortes-Gutiérrez E., MoralesVallarta M., Sampayo-Reyes A. \& Said-Fernández S. Activity of intracellular phospholipase A1 and A2 in Giardia lamblia. J. Parasitol., 2007, 93, 979-984.

WANG C.H., Su L.H. \& Sun C.H. A novel ARID/Bright-like protein involved in transcriptional activation of cyst wall protein 1 gene in Giardia lamblia.J. Biol. Chem., 2007, 282, 8905-8914.

World Health Organization (2006) World Health Report 2006, Geneve (Swiss).

Worgall T.S., Davis-Hayman S.R., Magana M.M., Oelkers P.M., Zapata F., Juliano R.A., Osborne T.F., Nash T.E. \& Deckelbaum R.J. Sterol and fatty acid regulatory pathways in a Giardia lamblia-derived promoter: evidence for SREBP as an ancient transcription factor. J. Lipid. Res., 2004, 45, 981-988.

Yellaturu C.R., Deng X., Cagen L.M., Wilcox H.G., Park E.A., RaghOW R. \& ELAM M.B. Posttranslational processing of SREBP-1 in rat hepatocytes is regulated by insulin and cAMP. Biochem. Biophys. Res. Commun., 2005, 332, 174180.

Reçu le 20 juin 2009

Accepté le 10 septembre 2009 